\title{
Individual and Combined Diagnostic Accuracy of Biochemical Markers for Detecting Early On-Set Preeclampsia
}

\author{
Samuel Asamoah Sakyi ${ }^{1 *}$, William K B A Owiredu ${ }^{1}$, Enoch 0 Anto ${ }^{1}$, Cornelius A Turpin ${ }^{2}$, \\ Richard K D Ephraim ${ }^{3}$ and Linda A Fondjo ${ }^{1}$ \\ ${ }^{1}$ Department of Molecular Medicine, School of Medical Sciences, Kwame Nkrumah University of Science and Technology, \\ Kumasi, Ghana \\ ${ }^{2}$ Department of Obstetrics \& Gynaecology, Komfo Anokye Teaching Hospital, Kumasi, Ghana \\ ${ }^{2}$ School of Medical Sciences, Kwame Nkrumah University of Science and Technology, Kumasi, Ghana \\ ${ }^{3}$ Department of Medical Laboratory Sciences, Faculty of Allied Health Sciences, College of Health and Allied Sciences, \\ University of Cape Coast, Cape Coast, Ghana
}

Received: June 20, 2016; Accepted: July 19, 2016; Published: July 29, 2016

*Corresponding author: Samuel Asamoah Sakyi, Department of Molecular Medicine, School of Medical Sciences, Kwame Nkrumah University of Science and Technology, Kumasi, Ghana; E-mail: samasamoahsakyi@yahoo.co.uk

\begin{abstract}
Background: Preeclampsia (PE) is one of the leading causes of maternal and neonatal mortality across the globe. Existing diagnostic parameters of PE have not proven to be sufficient in detecting the condition in its early stage. It is imperative to evaluate the biomarkers that are involve in the pathogenesis of PE, to identify which of them is specific and sensitive enough to detect early onset PE to prevent its associated adverse outcomes. This study evaluated the individual and combine diagnostic accuracy of angiogenic factors oxidative stress biomarkers, spot urine protein, creatinine and uric acid for detecting early onset PE.

Methods: A total of 165 pregnant women comprising of 110 women with PE and 55 pregnant women without PE (controls) were recruited from the Obstetrics and Gynaecology department at the Komfo Anokye Teaching Hospital (KATH). Blood samples were collected and assayed for Placental Growth Factor (PIGF), soluble fms-like tyrosine kinase 1 (sFlt-1) and 8-epi-Prostaglandin F2alpha (8-epi-PGF2 $\alpha$ ) levels using ELISA kits whilst total antioxidant capacity (T-AOC), urea, creatinine and uric acid were measured spectrophotometrically.

Results: Levels of PIGF, T-AOC, PIGF/sFlt-1 ratio, PIGF/8-epiPGF2 $\alpha$, and sFlt-1/8-epiPGF2 $\alpha$ were significantly reduced in early onset PE whilst sFlt-1, 8-epi-PGF2 $\alpha$, sFlt-1/PIGF ratio, 8-epiPGF2 $\alpha /$ PIGF, 8-epiPGF2 $\alpha /$ sFlt-1, spot urine protein/creatinine (Cr) ratio and Uric Acid (UA) were significantly increased in early-onset PE compared to late-onset PE $(p<0.05)$. In descending order, the most specific and sensitive biomarker for early onset PE were PIGF/sFlt-1 ratio $(0.81 ; 75.0 \%$ and $97.0 \% ; p<0.0001)$ followed by 8 -epiPGF2 $\alpha /$ PIGF $(0.73 ; 60.0 \%$ and $81.0 \% ; p=0.0020)$, sFlt-1/PIGF ratio $(0.79 ; 55.0 \%$ and $81.0 \% ; p<0.0001)$, PIGF/8-epiPGF2 $\alpha(0.71 ; 60.0 \%$ and $78.0 \% ; p=0.0010)$ and UA $(0.70 ; 50 \%$ and $79.0 \% ; p=$ 0.0340). At the various diagnostic cut-off of the markers, levels of PIGF, PIGF/sFlt-1, and PIGF/8-epiPGF2 $\alpha$ were reduced whilst elevated level of sFlt-1, sFlt-1/PIGF, and 8-epiPGF2 $\alpha /$ PIGF were significant predictors of early onset preeclampsia.
\end{abstract}

Conclusion: PIGF/sFlt-1 is a better diagnostic and predictive marker for early onset PE. Both early and late onsets PE were associated with alterations in various biochemical markers. Measurement of PIGF/sFlt-1 ratio should be included in pre-natal screening tests.

Keywords: Early-onset preeclampsia, diagnosis, biochemical markers, hypertension, proteinuria

\section{Abbreviations}

PE: Preeclampsia; UA: Uric acid; Cr: Creatinine; CHRPE: Committee on Human Research, Publications and Ethics; KATH: Komfo Anokye Teaching Hospital; PIGF: Placental growth factor; sFlt-1: Soluble fms-like tyrosine kinase 1; 8-epi-PGF2 $\alpha$ : 8-epiprostaglandin F2alpha; MDGs: Millennium Development Goals; VEGF-R1: Vascular endothelium growth factor receptor-1; T-AOC: Total antioxidant capacity.

\section{Introduction}

Preeclampsia (PE) is one of the leading cause of mother and neonate mortality that affects approximately 5-8\% of all pregnancies across the globe [1]. In recent times, several research efforts are been channeled to increase our understanding in the pathogenesis of PE. Early diagnosis and prevention of preterm delivery and its associated adverse complication are important in achieving Millennium Development Goals (MDGs) 4 and 5 [2]. Though medicinal intervention and various management strategies are in place to ameliorate hypertension in pregnancy [3], morbidity and mortality are still high. Till date, the only cure for PE is delivery of the placenta.

Routine diagnosis of PE is based on measurement of Blood 
Pressure (BP) and urine protein analysis coupled with clinical symptoms [4]. These measurements however, have not proven to be sufficient in diagnosing the condition in its early stage due to its low specificity with respect to prediction of the course of the disease as well as maternal and perinatal outcomes [4]. Uric acid (UA), has been found to promotes endothelial dysfunction and usually correlates well with the severity of the PE [5]. However, it is not a consistent predictive marker for early detection of PE, but generally increases once the disease manifest [5]. The diagnostic accuracy of spot urine protein and Creatinine (Cr) ratio and 24 hour urine protein excretion as a measure of proteinuria in PE have also been challenged [6,7]. Most studies [7,8] recommended the former over the latter whilst results from other studies are [9] inconsistent .

Increasing evidence suggest that imbalances in angiogenic factors and oxidative stress biomarkers may be the underlying cause of PE due to its involvement in placental development [10]. Endothelial dysfunction, the hallmark of PE originates from a reduced levels of Placental Growth Factors (PIGF) with corresponding increased Vascular Endothelium Growth Factor Receptor-1 (VEGF-R1) and pro-oxidants and thus may be used as diagnostic markers in predicting PE $[10,11]$. We have previously established that imbalance in the levels of angiogenic regulators and oxidative stress biomarkers correlates with adverse pregnancy outcomes among PE subjects. Hence, early identification of these imbalance would alert health care givers in anticipation of adverse pregnancy outcome and thus increased surveillance during pregnancy and parturition to ameliorate the adverse outcome [12].

Currently, there is no published data on the combined diagnostic accuracy of angiogenic factors and oxidative stress markers although some studies [13,14] explored the diagnostic performance of the individual biomarkers. The need to identify highly specific and sensitive biochemical markers is essential to aid in the diagnosis of early onset PE. It is against this background that this study evaluated the individual and the combine diagnostic accuracy of angiogenic factors and oxidative stress biomarkers as well as spot urine protein: creatinine ratio and uric acid for diagnosis of early onset PE.

\section{Materials and Methods}

\section{Study design/area}

This case-control study was carried out from August to December, 2015 at the Obstetrics and Gynaecology (O \& G) department of the KATH in the Ashanti Region of Ghana. The hospital has an average population of 4,780,380 (Ghana Statistical service, 2012). KATH is a thousand (1000) beds capacity and serves as a major referral centre for the middle belt and northern part of Ghana. The hospital also receives referrals from other regions and this gives fair representation of the Ghanaians population.

\section{Ethical approval and consent}

Ethical approval for this study was granted by the Committee on Human Research, Publications and Ethics (CHRPE), School of Medical Sciences, Kwame Nkrumah University of Science \& Technology (KNUST) and the Research and Development Committee of the KATH. Written informed consent in the form of a signature or fingerprint was obtained from all the participants prior to enrolment. It was clearly stated that participants were free to withdraw from the study at any time.

\section{Recruitment of participants}

A cohort of 165 pregnant women who had registered to access antenatal care at the obstetrics and Gynaecology department of the Komfo Anokye Teaching Hospital (KATH) were purposively recruited. During their periodic antenatal visits, 110 of them developed PE [58 in early onset ( $<34$ week) and 52 in late onset ( $>34$ week)], and 55 of them were normotensives and were used as controls. Participants were age-matched and the diagnosis of hypertensive disorders of pregnancy was done by qualified Obstetrician/Gynecologist using the National High Blood Pressure Education Program Working Group diagnostic criteria (2000). Information relating to obstetric and demographic characteristics was obtained using a self-structured closedended questionnaire. Information obtained from each subject was confirmed through record reviews of hospital database with a $100 \%$ rate of accuracy. Pregnant women both nulliparous and multiparous women aged 18-40 years, within the gestational age of $\geq 20$ - 40 weeks with singleton pregnancies were included in this study. Participants with twin pregnancy, previously diagnosed chronic hypertension, heart disease, gestational diabetic mellitus, gestational hypertension, use of antihypertensive medication before the recruitment as well as those who were unable to give informed consent were excluded from the study.

\section{Definition of clinical terms}

Preeclampsia was defined as the onset, after 20 weeks of gestation for both hypertension $(\geq 140 / 90 \mathrm{mmHg}$ ) and proteinuria of $>0.3 \mathrm{~g} / \mathrm{l}$ ( $\geq+1$ on dipstick) in two random urine samples collected at 4 to 6 hours apart. Participants with no hypertension and proteinuria after 20 week of gestation were consider as normal pregnant controls. Early onset preeclampsia was defined as PE before 34 completed weeks of gestation while late onset was considered as PE after 34 weeks of gestation [15].

\section{Blood Pressure measurements}

Blood pressure (BP) measurement was done by a trained personnel using mercury sphygmomanometer (Accoson, England) and a stethoscope in accordance with recommendations by the National High Blood Pressure Education Program Working Group diagnostic criteria [16]. The procedure was repeated two times for each patient between 5-10 minutes and the average BP values of duplicate measurements were recorded to the nearest $2.0 \mathrm{mmHg}$.

\section{Urine sample collection and estimation of proteinuria}

Approximately, $1 \times 10^{-5}-2 \times 10^{-5} \mathrm{~m}^{3}$ of freshly voided early morning urine were collected into clean, wide mouth and leak proof containers. Semi-quantitative proteinuria was immediately measured using dipstick (URIT 2V ${ }^{\mathrm{PG}}$. Jiuhua Road, Guilin, Guangxi 
541001, PR China). Proteinuria was defined as the presence of urinary protein in concentrations more than $0.3 \mathrm{~g} / \mathrm{l}$ or $1+$ on urine dipstick.

\section{Blood sample collection and Biochemical assays}

Six (6) $\times 10^{-5} \mathrm{~m}^{3}$ of venous blood sample was collected from each study participant. Blood was dispensed into serum separator gel containing vacutainer tubes and centrifuged (Nüve NF 200, Germany) at $3000 \mathrm{rpm}$ for 15 minutes. Serum was aliquoted and stored at $-80^{\circ} \mathrm{C}$ (Thermo Scientific ${ }^{\mathrm{TM}}$ Revco ${ }^{\mathrm{TM}} \mathrm{UxF}$-Ultra-Low Temperature Freezers, USA) until assay.

Serum levels of sFlt-1, PIGF and 8-epi-PGF2 $\alpha$ were measured in duplicate using commercially available ELISA kits from R\&D System Inc. (Minneapolis, MN USA). The optical density was measured at $450 \mathrm{~nm}$ using microplate ELISA reader (Mindray MR96A; Shenzhen Mindray Bio-medical electronics Co., Ltd, China). The plasma level of each factor was calculated using standard curves derived from a known concentration of the respective recombinant factors. Total Antioxidant Capacity (TAOC) reagents was obtained from Green stone Swiss Co., Ltd, China and serum levels were estimated spectrophotometrically (Mindray BA-88A; Shenzhen Bio-medical electronics Co., Ltd, China) at 593nm. This assay was measured based on the Ferric Reducing Ability of Plasma (FRAP) method as described by Benzie and Strain, (1999). All samples were analyzed in triplicate. Serum levels of urea, Creatinine (Cr), Blood Urea Nitrogen (BUN) and Uric Acid (UA) were measured spectrophotometrically using automated analyser (Mindray BA-88A; Shenzhen Bio-medical electronics Co., Ltd, China). PIGF/sFlt-1, sFlt-1/PIGF, 8-epiPGF2 $\alpha /$ PIGF, 8-epiPGF2 $\alpha$ /sFlt-1, PIGF/8-epiPGF2 $\alpha$, sFlt-1/8-epiPGF2 $\alpha$ and Spot urine protein: $\mathrm{Cr}$ ratios were calculated.

\section{Statistical analysis}

Statistical analysis was performed using XLSTAT 2014.5. The independent sample t-test was used to compare the two groups of parametric variables and Mann Whitney U test for nonparametric variables. Chi-square test for trend was used to test association between categorical variables. Data were expressed as mean \pm Standard Deviation (SD) for demographic continuous parametric data, as a frequency (percentage) for categorical data. Correlations were obtained by Pearson or Spearman where appropriate. The Receiver Operating Characteristics (ROC) curve was used to compute the Area Under the Curve (AUC) for each marker as well as Positive Predictive Values (PPVs), Negative Predictive Values (NPVs), likelihood ratios, threshold value, sensitivity, and specificity. Logistic regression analysis was performed to identify the predictive odds of the biomarker for early onset PE. Statistical significance was accepted at $\mathrm{p}<0.05$ for all comparisons.

\section{Results}

Demographic, obstetric and clinical characteristics of the studied participants are shown in Table 1 . The mean age of general participants was 29.78 years. There was no significant difference between the mean age of controls compared to preeclamptic women $(p=0.710)$. Mean Gestational Age (GA) at delivery was significantly lower in PE compared to controls $(p<0.001)$. Higher percentages (48.5\%) of the participants were nulliparous whilst $32.1 \%$ and $19.4 \%$ were multiparous and primiparous respectively. Most of the participants were primigravida (36.4\%) and higher percentage of them were preeclamptics $(43.6 \%)$ compared to control (21.8\%) ( $p=0.0041)$. The proportion of family history of hypertension $(24.5 \%$ vs $1.8 \% p<0.0001)$, and previous caesarean section $(21.8 \%$ vs $10.9 \% \mathrm{p}=0.0484)$ were higher in the PE compared to the controls. Contraceptive usage (7.3\% vs $27.3 \%$; $p=0.0012$ ) were significantly higher in the controls compared to the PE. Preeclamptics had significantly higher mean spot urine protein, Systolic Blood Pressure (SBP), Diastolic Blood Pressure (DBP), urea, Creatinine (Cr), Blood Urea Nitrogen Creatinine Ratio (BUN/Cr) compared to controls ( $p<$ 0.0001). Table 1

As shown in Figure 1, Participants with PE had significantly elevated levels of sFlt-1( $p<0.0001)$, sFlt-1/PIGF ratio ( $p<$ $0.0001)$ and 8-epi-PGF $2 \alpha(p<0.0001)$ and a reduced levels of PIGF ( $p<0.0001)$, PIGF/sFlt-1 ratio $(p<0.0001)$ and T-AOC $(p<$ $0.0001)$ compared to controls Figure 1.

Levels of angiogenic and oxidative stress markers in early and late onset PE are shown in Figure 2. There were elevated levels of sFlt-1 ( $p=0.0421)$, sFlt-1/PIGF ratio $(p=0.0485)$ and 8-epiPGF $2 \alpha(p=0.0121)$ and a reduced levels of PIGF $(p<0.0001)$, PIGF/sFlt-1 ratio $(p=0.0071)$ and T-AOC $(p=0.0471)$ in early onset PE compared to late onset Figure 2.

As shown in Figure 3, levels of uric acid ( $p=0.0590)$, spot urine protein: $\mathrm{Cr}$ ratio $(p=0.1363)$, 8-epiPGF2 $\alpha /$ PIGF $(p=0.0190)$, and 8-epiPGF $2 \alpha /$ sFlt-1 $(p=0.0590)$ were higher while PIGF/8epiPGF $2 \alpha(p=0.0695)$, and sFlt-1/8-epiPGF2 $\alpha(p=0.0942)$ were lower in early onset compared to late onset PE Figure 3.

Analysis on Spearman rho moment correlation indicated that a statistically significant $(p<0.05)$ negative correlation of BP (SBP and DBP), UA, spot urine protein: Cr, parity and BMI was observed with PIGF, T-AOC and PIGF/sFlt-1 ratio compared while a statistically significant positive correlation was observed with sFlt-1, 8-epi-PGF2 $\alpha$, and sFlt-1/PIGF ratio $(p<0.05)$. The correlations of angiogenic factors and oxidative stress markers with BP (SBP and DBP) and spot urine protein: Cr ratio was significant $(p<0.05)$ after adjusting for age, BMI and parity. (Table 2) UA correlated significantly with oxidative stress biomarkers after adjusting for age, BMI and parity not angiogenic factors Table 2. Figure 4, Figure 5, Figure 6.

Table 3 shows the sensitivity and specificity pattern of angiogenic factors, oxidative stress biomarkers, UA and spot urine protein: $\mathrm{Cr}$ ratio. The diagnostic thresholds were $14.30 \mathrm{pg} /$ $\mathrm{ml}$ for PIGF, $838.5 \mathrm{pg} / \mathrm{ml}$ for sFlt-1, $404.30 \mathrm{pg} / \mathrm{ml}$ for 8-epiPGF2 $\alpha$, $0.38 \mathrm{mmol} / \mathrm{l}$ for T-AOC, 18.00 for sFlt-1/PIGF ratio, 0.60 for PIGF/ sFlt-1 ratio, 7.2 for 8-epiPGF2 $\alpha /$ IIGF, 0.48 for 8 -epiPGF $2 \alpha /$ sFlt1, 0.14 for PIGF/8-epiPGF2 $\alpha, 1.13$ for sFlt-1/8-epiPGF $2 \alpha, 11.60$ for spot-urine protein: $\mathrm{Cr}$ and $440.00 \mathrm{umol} / \mathrm{l}$ for UA. However, the most accurate, specific and sensitive marker was PIGF/sFlt-1 ratio $(0.81 ; 75.0 \%$ and $97.0 \% ; p<0.0001)$ followed by sFlt-1/ PIGF ratio (0.79; 81.0\% and 55.0\%; $p<0.0001$ ), 8-epiPGF $2 \alpha$ / 
Table 1: Demographic, obstetrics, clinical and biochemical characteristic of participants.

\begin{tabular}{|c|c|c|c|c|}
\hline Variables & $\begin{array}{l}\text { Total } \\
(n=165)\end{array}$ & Controls $(n=55)$ & $\begin{array}{c}\text { PE } \\
(n=110)\end{array}$ & p-value \\
\hline Age (years) & $29.78 \pm 0.40$ & $29.99 \pm 0.44$ & $29.85 \pm 0.53$ & 0.710 \\
\hline Gestational age (weeks) & $36.12 \pm 0.71$ & $38.09 \pm 0.37$ & $36.02 \pm 0.27$ & $<0.0001$ \\
\hline Parity & & & & 0.9455 \\
\hline Nulliparous & $80(48.5 \%)$ & $25(45.5 \%)$ & $55(50.0 \%)$ & \\
\hline Primiparous & $32(19.4 \%)$ & $12(21.8 \%)$ & $20(18.2 \%)$ & \\
\hline Multiparous & $53(32.1 \%)$ & $18(32.7 \%)$ & $35(31.9 \%)$ & \\
\hline Gravidity & & & & 0.0041 \\
\hline Primigravida & $60(36.4 \%)$ & $12(21.8 \%)$ & $48(43.6 \%)$ & \\
\hline Secundigravida & $57(34.5 \%)$ & $17(30.9 \%)$ & $40(36.4 \%)$ & \\
\hline Multigravida & $48(29.0 \%)$ & $26(47.3 \%)$ & $22(20.0 \%)$ & \\
\hline Economic income (GHS) & & & & 0.0013 \\
\hline$<500$ GHS (low income) & $134(81.2 \%)$ & $33(60.0 \%)$ & 101(91.8\%) & \\
\hline 500-1000 GHS (middle income) & $26(15.8 \%)$ & $18(32.7 \%)$ & $8(7.3 \%)$ & \\
\hline$>1000$ (high income) & $5(3.0 \%)$ & $4(7.3 \%)$ & $1(0.9 \%)$ & \\
\hline \multicolumn{5}{|l|}{ Family history of HTN } \\
\hline Yes & $28(16.9 \%)$ & $1(1.8 \%)$ & $27(24.5 \%)$ & $<0.0001$ \\
\hline \multicolumn{5}{|l|}{ Previous Caesarean section } \\
\hline Yes & $30(18.2 \%)$ & $6(10.9 \%)$ & $24(21.8 \%)$ & 0.0484 \\
\hline Early gestation BMI $\left(\mathrm{Kg} / \mathrm{m}^{2}\right)$ & $23.49 \pm 5.52$ & $21.07 \pm 7.53$ & $25.90 \pm 6.51$ & 0.0181 \\
\hline SBP (mmHg) & $139.75 \pm 1.32$ & $114.30 \pm 0.99$ & $165.20 \pm 1.64$ & $<0.0001$ \\
\hline DBP (mmHg) & $88.97 \pm 1.04$ & $69.33 \pm 0.96$ & $108.60 \pm 1.12$ & $<0.0001$ \\
\hline Urea (mmol/L) & $4.03 \pm 0.40$ & $1.99 \pm 0.11$ & $6.07 \pm 0.69$ & $<0.0001$ \\
\hline $\mathrm{Cr}(\mathrm{umol} / \mathrm{l})$ & $84.48 \pm 10.53$ & $54.77 \pm 1.77$ & $114.2 \pm 19.30$ & 0.0284 \\
\hline $\mathrm{BUN} / \mathrm{Cr}$ & $17.67 \pm 0.78$ & $11.25 \pm 0.23$ & $24.08 \pm 1.32$ & $<0.0001$ \\
\hline Uric acid (umol/l) & $352.3 \pm 0.11$ & $303.0 \pm 7.37$ & $401.7 \pm 9.85$ & $<0.0001$ \\
\hline Spot urine protein (g/l) & $1.03 \pm 0.06$ & $0.01 \pm 0.00$ & $2.05 \pm 0.11$ & $<0.0001$ \\
\hline
\end{tabular}

Values are presented as frequency (proportion) and Mean $\pm S D .{ }^{*} p<0.05,{ }^{* *} p<0.001,{ }^{* * *} p<0.0001$ is considered statistically significance difference. HTN: Hypertension; ANT: Antenatal; GA: Gestational age; SBP: systolic blood pressure; DBP: diastolic blood pressure
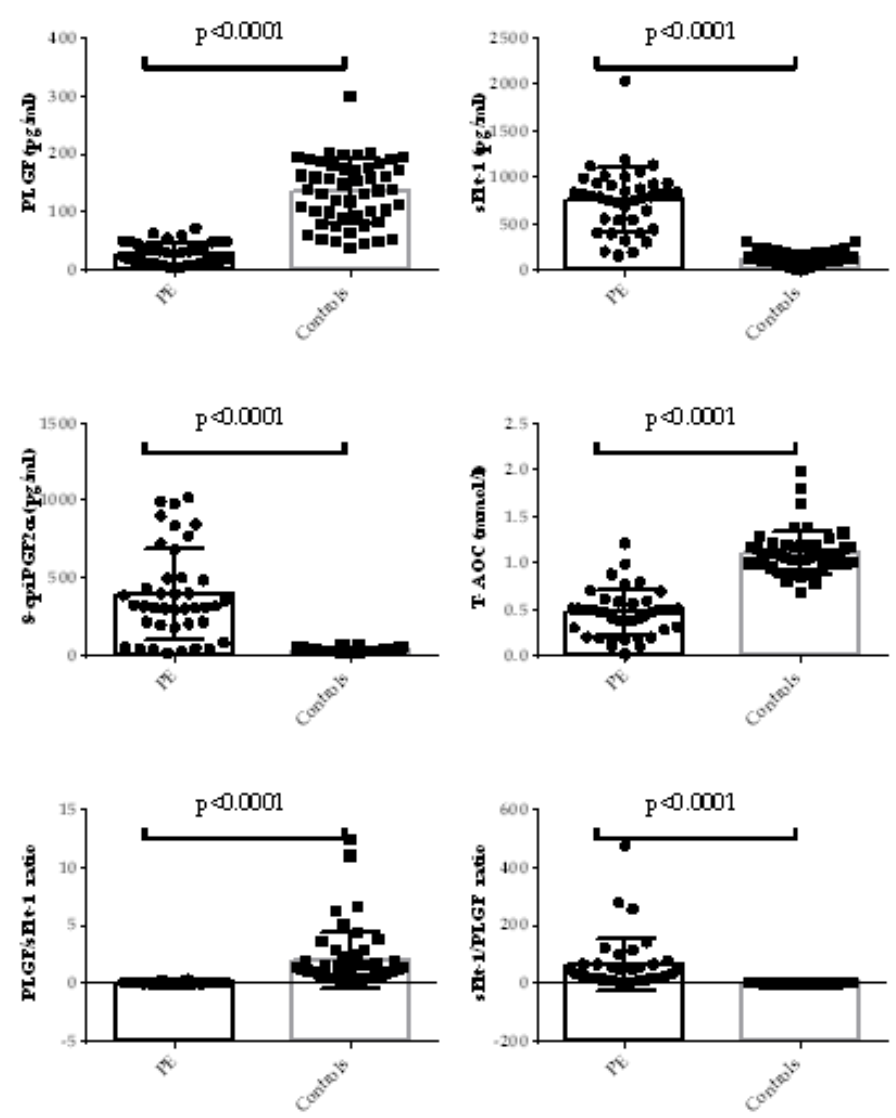

Figure 1: Levels of angiogenic factors and oxidative biomarkers levels in PE and normotensive pregnant women. Scatter dot plots compare early-onset ( $<34$ weeks of gestation) to late-onset ( $>34$ weeks of gestation). $p<0.05$ was considered statistically significant. 

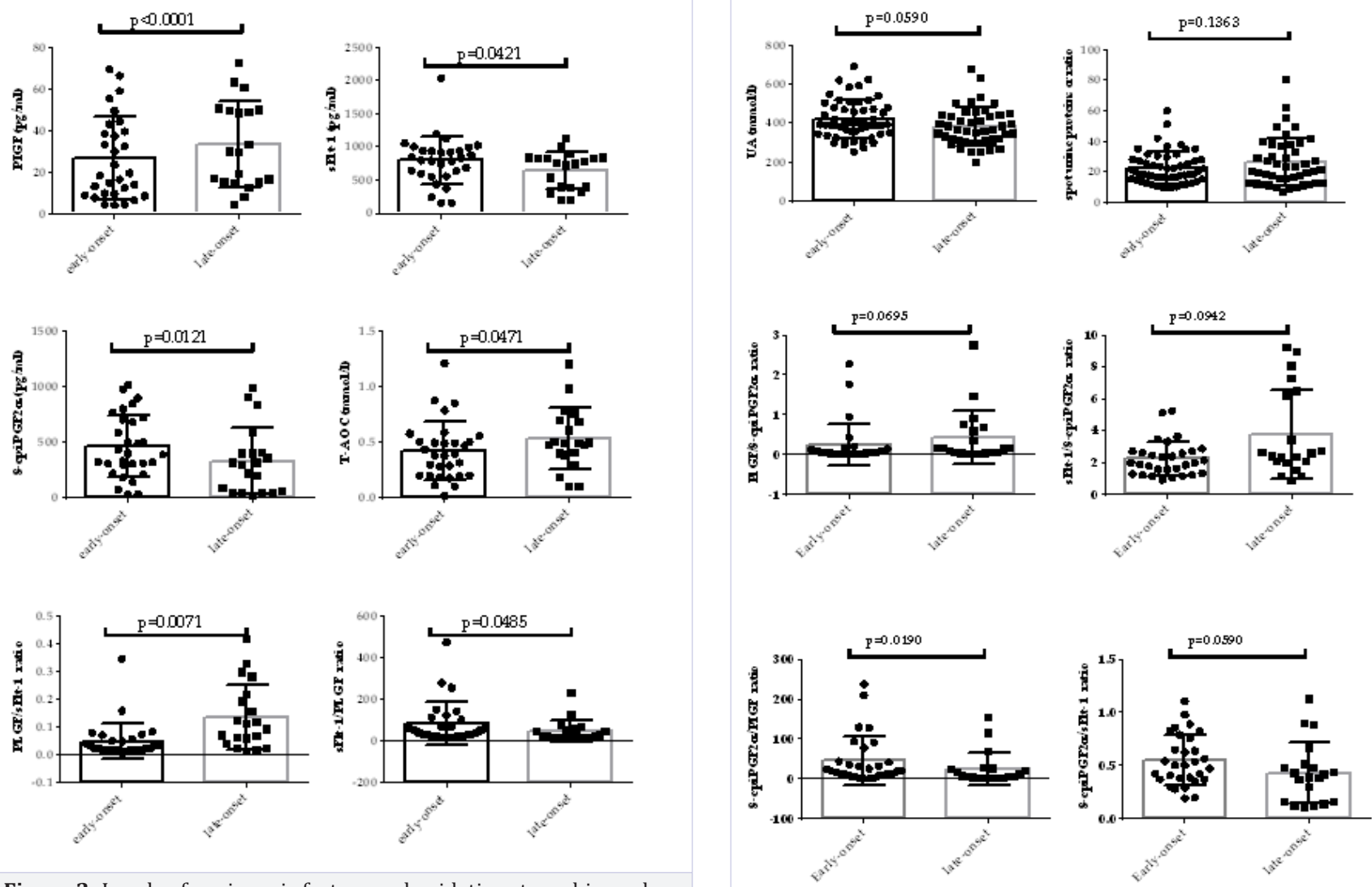

Figure 2: Levels of angiogenic factors and oxidative stress biomarkers in early and late onset preeclampsia. Scatter dot plots compares earlyonset ( $<34$ weeks of gestation) to late-onset ( $>34$ weeks of gestation).

Figure 3: Levels of urine uric acid (UA), spot urine protein: $\mathrm{Cr}$ ratio, the ratio of 8-epiPGF2 $\alpha / \mathrm{PIGF}$, 8-epiPGF2 $\alpha /$ sFlt-1, PIGF/8-epiPGF2 $\alpha$, sFlt$1 / 8$-epiPGF2 $\alpha$ in early and late onset preeclampsia

Table 2: Bivariate and Partial correlation of angiogenic and oxidative biomarkers with BP, GA, UA, spot urine protein: Cr ratio after adjusting for maternal age, parity, BMI in early onset PE.

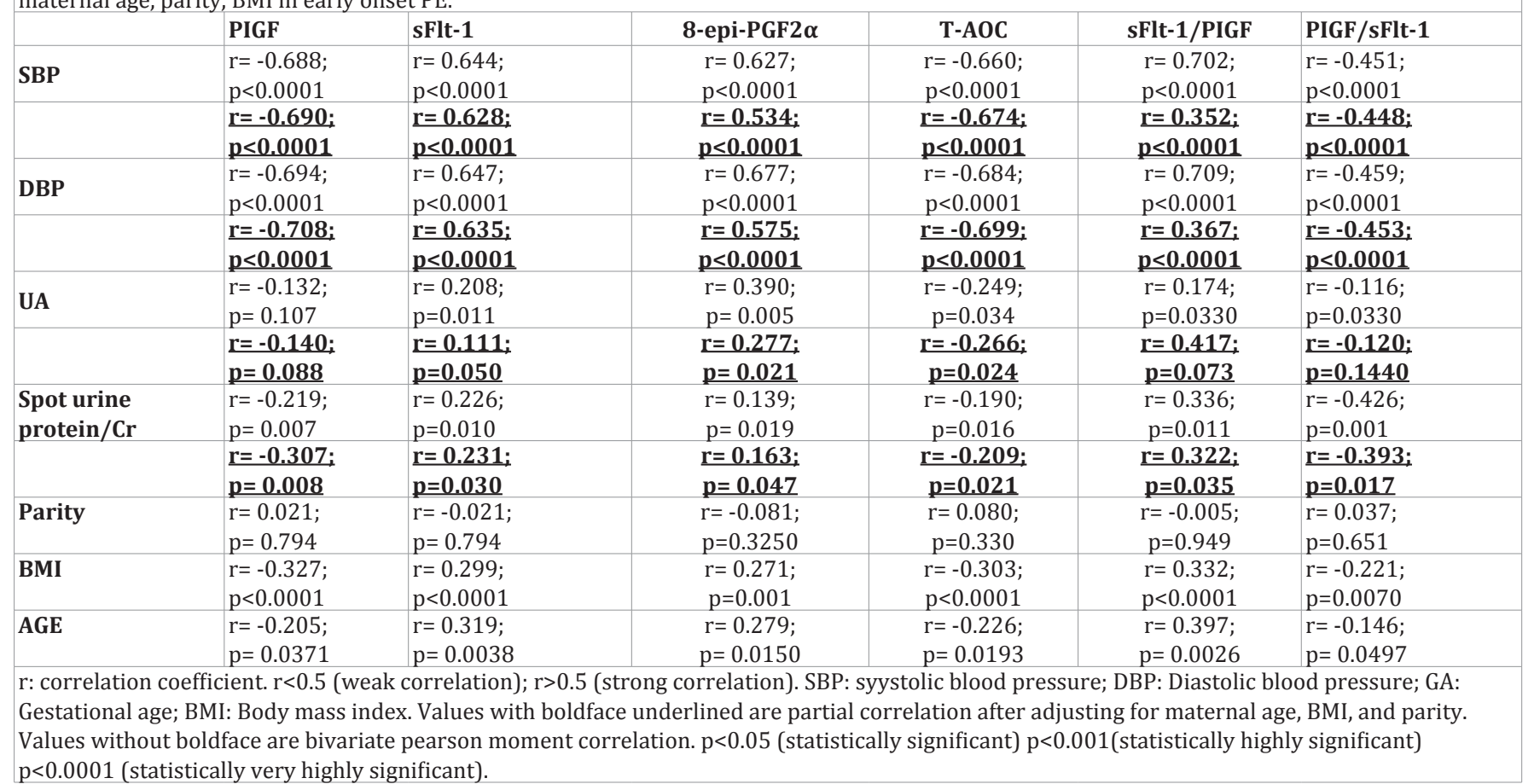




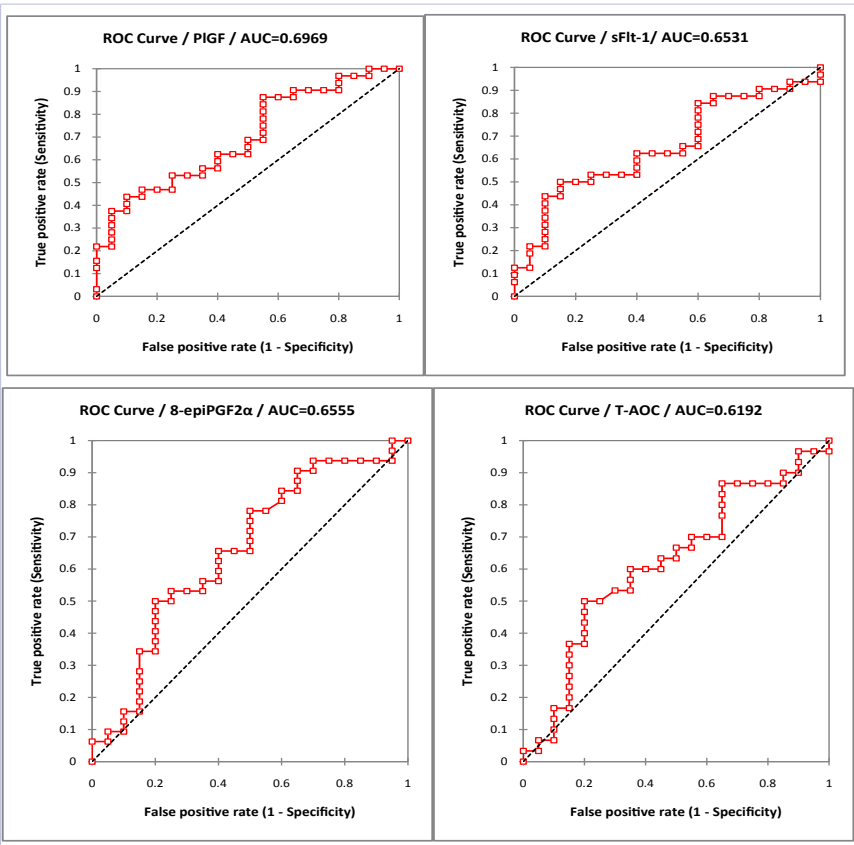

Figure 4: Receivers operating Characteristics (ROC) curve of PIGF, sFlt1,8 -epi-PGF2 $\alpha$ and T-AOC showing area under the curve (AUC).

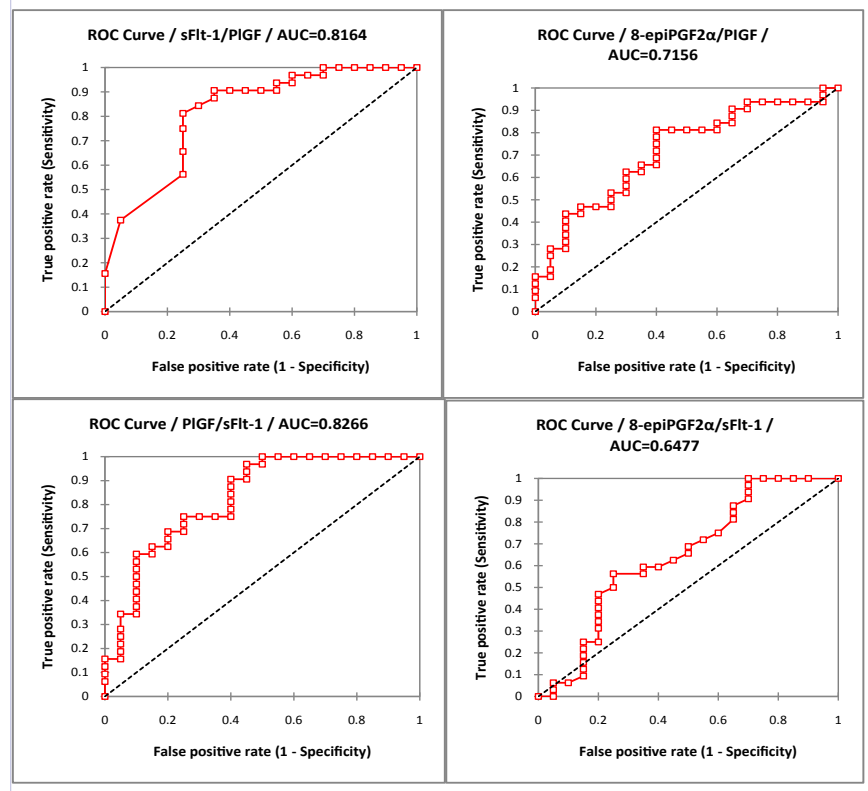

Figure 5: Receivers operating Characteristics (ROC) curve of PIGF/ sFlt-1, sFlt-1/PIGF, 8-epi-PGF2 $\alpha /$ PIGF and 8-epi-PGF2 $\alpha /$ sFlt-1 ratio showing area under the curve (AUC).

PIGF $(0.73 ; 60.0 \%$ and $81.0 \% ; p=0.0020)$, PIGF/8-epiPGF2 $\alpha$ $(0.71 ; 60.0 \%$ and $78.0 \% ; p=0.0010)$ and UA $(0.70 ; 50 \%$ and $79.0 \% ; p=0.0340)$. PIGF $(90.0 \%)$ was the most specific followed by sFlt-1(85.0\%), 8-epiPGF2 $\alpha(80.0 \%)$ and T-AOC $(80.0 \%)$ but had a poor sensitivity ( $44.0 \%$, followed by $50.0 \%, 50.0 \%, 50.0 \%$ ). Spot urine protein: $\mathrm{Cr}$ ratio and sFlt-1/8-epiPGF $2 \alpha$ were very sensitive but had poor specificity Table 3 .
Table 4 shows the logistic regression of biomarkers for early onset preeclampsia. After adjusting for age, early gestation BMI and parity, PIGF levels $<14.3 \mathrm{pg} / \mathrm{ml}$ was significantly ( $p=0.0135$ ) associated with 7 times increase odds, sFlt-1 levels $>838.5 \mathrm{pg} /$ $\mathrm{ml}$ was significantly ( $p=0.0309$ ) associated 1.61 times increase odds, sFlt-1/PIGF ratio $>18.0$ was significantly $(p=0.002)$ associated with 2.96 times increase odds, PIGF/sFlt-1 ratio $<0.60$ was significantly $(p<0.0001)$ associated with 35.08 times increase odds, 8 -epiPGF2 $\alpha /$ PIGF ratio $>7.2$ was significantly ( $p$ $=0.009$ ) associated with 1.74 times increase odds, and PIGF/8epiPGF $2 \alpha$ ratio $<0.14$ was significantly ( $p=0.0212$ ) associated with 1.61 times increase odds for early onset PE Table 4.

\section{Discussion}

This study evaluated the diagnostic performance and predictive ability of angiogenic factors, oxidative stress, uric acid and spot urine protein: creatinine ratio for early onset $\mathrm{PE}$. Individually, sFlt-1/PIGF, sFlt-1, 8-epiPGF2 $\alpha$, UA and spot urine protein: $\mathrm{Cr}$ ratio were significantly increased with decreases in PIGF, PIGF/sFlt-1 and T-AOC levels in PE compared to normotensive pregnant women as well as early onset compared to late onset preeclampsia. PIGF/sFlt-1 ratio proved to be the most accurate, specific and sensitive marker for early onset preeclampsia. This finding is consistent with a current prospective observational study which concluded that sFlt-1: PIGF ratio of 38 or lower can be used to predict the short-term absence of preeclampsia in women in whom the syndrome is suspected clinically [21]. Saleh et al., also observed that an elevated ratio is superior to the clinical diagnosis of PE for predicting an adverse pregnancy outcome and a low ratio is inversely correlated with prolongation of pregnancy [22].

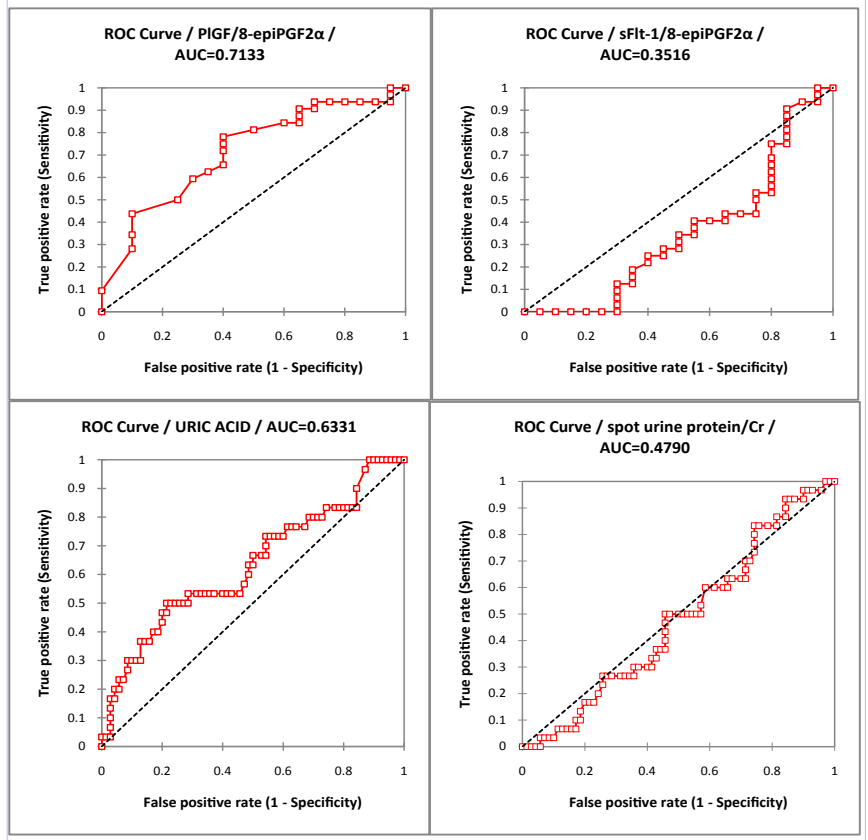

Figure 6: Receivers operating Characteristics (ROC) curve of PIGF/8epi-PGF2 $\alpha$, sFlt-1/8-epi-PGF2 $\alpha$, spot urine protein: $\mathrm{Cr}$ ratio and UA showing area under the curve (AUC). 
Table 3: Diagnostic Performance of angiogenic factors, oxidative stress biomarkers, spot urine protein: $\mathrm{Cr}$ and UA in predicting early onset of preeclampsia.

\begin{tabular}{|c|c|c|c|c|c|c|c|c|c|}
\hline Biomarkers & Threshold value & Sensitivity $(95 \% \mathrm{CI})$ & Specificity $(95 \% \mathrm{CI})$ & PPV & NPV & LR+ & LR- & $\begin{array}{c}\text { Diagnostic } \\
\text { Accuracy }\end{array}$ & p-value \\
\hline PIGF & $14.30 \mathrm{pg} / \mathrm{ml}$ & $0.44(0.28-0.61)$ & $0.90(0.68-0.98)$ & 0.88 & 0.50 & 4.38 & 0.63 & 0.62 & 0.0050 \\
\hline sFlt-1 & $838.50 \mathrm{pg} / \mathrm{ml}$ & $0.50(0.34-0.66)$ & $0.85(0.63-0.95)$ & 0.84 & 0.52 & 3.33 & 0.59 & 0.64 & 0.0390 \\
\hline 8-epiPGF2 $\alpha$ & $404.30 \mathrm{pg} / \mathrm{ml}$ & $0.50(0.34-0.66)$ & $0.80(0.58-0.92)$ & 0.80 & 0.50 & 2.50 & 0.63 & 0.62 & 0.0470 \\
\hline T-AOC & $0.38 \mathrm{mmol} / \mathrm{l}$ & $0.50(0.33-0.67)$ & $0.80(0.57-0.92)$ & 0.79 & 0.52 & 2.50 & 0.63 & 0.62 & 0.1350 \\
\hline sFlt-1/PIGF & 18.00 & $0.81(0.64-0.91)$ & $0.65(0.34-0.74)$ & 0.78 & 0.71 & 2.15 & 0.06 & 0.79 & $<0.0001$ \\
\hline PIGF/sFlt-1 & 0.60 & $0.97(0.82-1.00)$ & $0.75(0.53-0.89)$ & 0.84 & 0.92 & 3.25 & 0.25 & 0.81 & $<0.0001$ \\
\hline 8-epiPGF2 $\alpha /$ PIGF & 7.20 & $0.81(0.64-0.91)$ & $0.60(0.39-0.78)$ & 0.76 & 0.67 & 2.03 & 0.31 & 0.73 & 0.0020 \\
\hline 8-epiPGF2 $\alpha /$ sFlt-1 & 0.48 & $0.56(0.39-0.72)$ & $0.75(0.53-0.89)$ & 0.78 & 0.52 & 2.25 & 0.58 & 0.63 & 0.0690 \\
\hline PIGF/8-epiPGF2 $\alpha$ & 0.14 & $0.78(0.60-0.89)$ & $0.60(0.39-0.78)$ & 0.76 & 0.63 & 1.95 & 0.36 & 0.71 & 0.0010 \\
\hline sFlt-1/8-epiPGF2 $\alpha$ & 1.13 & $0.91(0.75-0.97)$ & $0.15(0.05-0.37)$ & 0.63 & 0.50 & 1.07 & 0.63 & 0.62 & 0.0680 \\
\hline Spot-urine protein: $\mathrm{Cr}$ & 11.60 & $0.93(0.77-0.99)$ & $0.16(0.09-0.26)$ & 0.32 & 0.85 & 1.11 & 0.42 & 0.39 & 0.7260 \\
\hline UA & $440.00 \mathrm{umol} / \mathrm{l}$ & $0.50(0.33-0.67)$ & $0.79(0.67-0.86)$ & 0.50 & 0.79 & 2.33 & 0.64 & 0.70 & 0.0340 \\
\hline
\end{tabular}

PPV: positive predictive value; NPV: negative predictive value; CI: confidence interval; AUC: Area under the curve; LR+: Positive likelihood ratio; LR-: Negative likelihood ratio; UA: Uric acid; BUN: Cr: blood urea nitrogen: creatinine ratio; PIGF: Placental growth factor; sFlt-1: soluble fms-like tyrosine kinase; 8-epiPGF2a: 8-epipostaglandin F2-alpha

Table 4: Multivariate Logistic regression analysis of predictive ability of biomarkers for early onset preeclampsia.

\begin{tabular}{|l|c|c|c|}
\hline Biomarkers & Threshold value & A0R (95\% CI) & p-value \\
\hline PIGF & $<14.3 \mathrm{pg} / \mathrm{ml}$ & $7.00(1.386$ to 35.36$)$ & 0.0135 \\
\hline sFlt-1 & $>838.5 \mathrm{pg} / \mathrm{ml}$ & $1.61(1.031-2.517)$ & 0.0309 \\
\hline 8-epiPGF2 $\alpha$ & $>404.3 \mathrm{pg} / \mathrm{ml}$ & $0.68(0.446-1.045)$ & 0.0792 \\
\hline T-AOC & $<0.38 \mathrm{mmol} / \mathrm{l}$ & $0.81(0.701-0.984)$ & 0.0918 \\
\hline sFlt-1/PIGF & $>18.0$ & $2.69(1.438-5.053)$ & 0.0020 \\
\hline PIGF/sFlt-1 & $<0.60$ & $35.08(24.83-42.08)$ & $<0.0001$ \\
\hline 8-epiPGF2 $\alpha /$ PIGF & $>7.2$ & $1.74(1.148-2.632)$ & 0.0090 \\
\hline 8-epiPGF2 $\alpha /$ sFlt-1 & $>0.48$ & $0.74(0.489-1.105)$ & 0.1394 \\
\hline PIGF/8-epiPGF2 $\alpha$ & $<0.14$ & $1.61(1.074-2.425)$ & 0.0212 \\
\hline sFlt-1/8-epiPGF2 $\alpha$ & $>1.13$ & $1.10(0.748$ to 1.619$)$ & 0.6240 \\
\hline Spot urine protein : Cr ratio & $>1.60$ & $0.501(0.325-0.817)$ & 0.0718 \\
\hline UA & $>440 \mu$ mol/l & $1.001(0.812-1.215)$ & 0.0514 \\
\hline AOR: adjusted Odds ratio; Cl: confidence interval. Age, BMI and parity adjusted odds ratio. & & \\
\hline
\end{tabular}

In another observational prospective study by De Vivo. et al, [13] among women from Messina and a cross-sectional study by Pinheiro. et al, [17] among Brazilian women. The antagonistic activity of sFlt-1 on PIGF action may have led to the angiogenic imbalance culminating in the dysfunction of the endothelium and it integrity. This study also reported significantly higher levels of 8-epiPGF $2 \alpha$ and a lower T-AOC concentration in the early onset compared to late onset. These findings are consistent with case-control study by Wikström. et al, [18] among pregnant women from Sweden who observed significantly higher 8-isoprostaglandin in early onset compared to late onset PE. The current study further observed that spot urine protein: $\mathrm{Cr}$ ratio was higher in early onset compared to late onset, this findings is consistent with studies by Côté. et al, [8] though there were no statistically significant difference. The current study indicate that PIGF/sFlt- 1 ratio is the most accurate (AUC=0.82), with sensitivity of $97.0 \%$ and specificity of $75.0 \%$ with a PPV of $84.0 \%$ and NPV of $(92.0 \%$ at a threshold 0.60 for early onset preeclampsia (Figure 5). The current study shows that PIGF/sFlt-1 ratio is the most accurate maker in early onset $\mathrm{PE}(<34$ week gestation). Below the threshold value of 0.60 for PIGF/sFlt- 1 ratio, PE patients were 35.08 times more likely to develop early onset PE (Table 4) than late onset indicating that this marker is an important diagnostic and predictive tool. PIGF/sFlt-1 ratio being strongly associated with early onset PE reflects the modified balance between sFlt1 and PIGF. However, large sample population coupled with a prospective cohort study is needed to confirm this finding.

This case-control study evaluated the diagnostic accuracy of sFlt-1/PIGF in the second and third trimester of pregnancy. This study observed that using sFlt-1/PIGF proved to be sensitive $(81.0 \%)$ with PPV of $78.0 \%$ and NPV of $71.0 \%$ but was associated with poor specificity (55.0\%). Above the threshold value of 18.0 
for sFlt-1/PIGF, PE patients were 2.69 times more likely to develop early onset $\mathrm{PE}$. The current study for the first time identified the ratios of 8-epiPGF2 $\alpha /$ PIGF and PIGF/8-epiPGF2 $\alpha$ as significant diagnostic markers for early onset PE with sensitivity (81.0\% vs $78 \%$ ), specificity ( $60.0 \%$ vs $60.0 \%)$, PPV (76\% vs $76 \%$ ) and NPV (67\% vs $63 \%$ ). At a threshold of 7.2 and above for 8 -epiPGF $2 \alpha$ / PIGF ratio and 0.14 and below for PIGF/8-epiPGF2 $\alpha$, PE patient are 1.74 times and 1.61 times respectively more likely to develop early onset PE. 8-epiPGF2 $\alpha /$ PIGF ratio gave a better diagnostic value for early onset PE compared to previously known sFlt-1/ PIGF ratio. However, at the threshold value sFlt-1/PIGF ratio was more likely to predict early onset PE compared to 8-epiPGF2 $\alpha$ / PIGF ratio (2.69 times vs 1.74 times). The combine effect of angiogenic factors and oxidative stress biomarkers indicates the synergic role they play in the pathogenesis of PE. Further studies are therefore needed to prove the diagnostic potency of 8-epiPGF2 $\alpha /$ PIGF.

The individual markers of angiogenic factor (PIGF and sFlt1) and oxidative stress biomarker (8-epiPGF2 $\alpha$ and T-AOC) proved to be highly specific but poorly sensitive (Table 3 ) thus, their usefulness in early onset PE may be unreliable. The onset threshold levels for sFlt-1 are relatively higher in earlier gestations and begins to deviate from the reference range in preeclamptic patients suggesting their low levels in early onset preeclampsia [19]. Below $<14.3 \mathrm{pg} / \mathrm{ml}$ of PIGF, PE patients are 7.0 times more likely to develop early onset while sFlt-1 levels $>838.5 \mathrm{pg} / \mathrm{ml}$ was associated with 1.61 times increase odds. The finding indicates that PIGF can be considered as better predictor for early than sFlt-1. This finding is consistent with a study conducted by Ohkuchi. et al, [20]. This outcome may explain the role PIGF plays in the pathogenic process in the development of preeclampsia. Based on these findings it may be suggested that, sFlt-1 should be considered as a late marker of pre-eclampsia than an early onset marker.

This study also observed that spot urine protein: $\mathrm{Cr}$ proved to be highly sensitive (93.0\%) but lack specificity (16.0\%) with poor diagnostic accuracy (0.39). A recent study by Baba. et al, among normotensive pregnant women showed a significant correlation between the Protein/Cr ratio and 24-h urine protein level [23]. Other previous studies $[4,6,8]$ accredit spot urine protein: $\mathrm{Cr}$ to be better marker for identifying proteinuria in PE. However, in this study, spot urine protein: $\mathrm{Cr}$ ratio was 50\% less likely to predict early onset PE [aOR $=0.501(0.325-0.817)]$. This result suggest that using spot urine protein: $\mathrm{Cr}$ ratio could identify any form of proteinuria related condition but lacks specificity to specific proteinuria condition. Thus incorporating this marker in routine maternal and fetal investigation will only be useful to identify proteinuria and thus not a better marker for early onset PE. However, further studies may be needed to explore it usefulness.

Using UA as a diagnostic tool and predictive factor for the development of pre-eclampsia, at a significant threshold value of $440 \mu \mathrm{mol} / \mathrm{l}$, the sensitivity, specificity, PPV, NPV and diagnostic accuracy were $50.0 \%, 79 \%, 50.0 \%, 79.0 \%$ and 0.70 respectively. However UA may not be a better predictive tool because at levels greater than threshold value it is indecisive [ $(\mathrm{aOR}=1.001(0.812$ 1.215)] to predict early onset PE. Previous study indicates that plasma levels of UA usually increase once the disease manifests and it's more likely to correlate with disease severity. UA correlated significantly with oxidative stress biomarkers but not angiogenic factors indicating that mechanism of elevated UA in PE does not act through the angiogenic pathway.

The main limitation of the current study is the inability to conduct a longitudinal cohort study which could have assessed the changes over time, however, findings from this study will serve as a baseline for further studies to address this interest.

\section{Conclusion}

All the biomarkers evaluated play significant roles in the onset of preeclampsia and can therefore be used as biomarkers for diagnosing PE. However, the PIGF/s-Flt1 ratio proved to be a most accurate and predictive marker for early onset PE. Angiogenic factors, oxidative stress biomarkers, spot urine: $\mathrm{Cr}$ ratio and UA are altered in early onset PE compared to late onset. The combine diagnostic performance of angiogenic factors and oxidative stress biomarkers poses a better diagnostic accuracy, sensitivity and specificity while individual markers were highly specific with poor sensitivity for early onset PE. Measurement of PIGF/sFlt-1 ratio should be included in pre-natal screening tests.

\section{Declarations}

\section{Ethical approval and consent}

Ethical approval for this study was granted by the Committee on Human Research, Publications and Ethics (CHRPE), School of Medical Science, Kwame Nkrumah University of Science \& Technology (KNUST) and the Research and Development Committee of the KATH. Written informed consent in the form of a signature or fingerprint was obtained from all the participants prior to enrolment. It was clearly stated that participants were free to withdraw from the study at any time.

\section{Consent to publish}

All persons have done sufficient work to justify authorship for this article and has approve that the manuscript be published

\section{Authors' contributions}

Conceived and designed the experiments: Samuel Asamoah Sakyi, Enoch O Anto, Cornelius A Turpin, William K B A Owiredu, and Richard K D Ephraim.

Performed the experiments: Enoch O Anto, William K B A Owiredu, Samuel Asamoah Sakyi, Linda A Fondjo and Cornelius A Turpin.

Analyzed the data: Enoch 0 Anto, Linda A Fondjo, and Richard K D Ephraim

Wrote the first draft of the manuscript: Enoch O Anto, Samuel Asamoah Sakyi, and William K B A Owiredu.

Contributed to the writing of the manuscript: Enoch 0 Anto, Samuel Asamoah Sakyi, William K B A Owiredu, Cornelius A 


\section{Turpin and Richard K D Ephraim.}

Agree with manuscript results and conclusions: Enoch 0 Anto, Samuel Asamoah Sakyi, Linda A Fondjo, WKBAO and Richard K D Ephraim.

Enrolled Patients: Enoch O Anto, Samuel Asamoah Sakyi, Linda A Fondjo and Cornelius A Turpin. All authors read and approved the final manuscript.

\section{Competing interest}

The authors of this manuscript declare that there is no competing interest

\section{Acknowledgement}

The authors would like to acknowledge the Department of O\&G, KATH and Department of Molecular medicine and serology department of KATH. We also wish to thank the Midwives for their immense support during sample collection. We also would like to thank the Medilab diagnostic centre, Kumasi for they help in biochemical analysis. We wish to thank the study participants

\section{Availability of data and materials}

All relevant raw data, will be made freely available to any scientist wishing to use them for non-commercial purposes, moreover, the dataset supporting the conclusions of this article are included within the article.

\section{References}

1. Walker JJ. Pre-eclampsia. Lancet. 2000;356(9237):1260-1265.

2. WHO: Maternal mortality in 1995: Estimates developed by WHO, UNICEF and UNFPA: WHO Geneva; 2004.

3. Wood AJ, Sibai BM. Treatment of hypertension in pregnant women. New England Journal of Medicine. 1996;335(4):257-265.

4. Sethuram R, Kiran TSU, Weerakkody AA. Is the urine spot protein/ creatinine ratio a valid diagnostic test for pre-eclampsia?. Journal of Obstetrics \& Gynaecology. 2011;31(2):128-130.

5. Martin AC, Brown MA. Could uric acid have a pathogenic role in preeclampsia?. Nature reviews nephrology. 2010;6(12):744-748.

6. Al RA, Baykal C, Karacay O, Geyik PO, Altun S, Dolen I. Random urine protein-creatinine ratio to predict proteinuria in new-onset mild hypertension in late pregnancy. Obstetrics \& Gynecology. 2004;104(2):367-371.

7. Shahbazian N, Hosseini-Asl F. A comparison of spot urine proteincreatinine ratio with 24-hour urine protein excretion in women with preeclampsia. Iran J Kidney Dis. 2008;2(3):127-131.

8. Côté AM, Brown MA, Lam E, von Dadelszen P, Firoz T, Liston RM, et al. Diagnostic accuracy of urinary spot protein: creatinine ratio for proteinuria in hypertensive pregnant women: systematic review. Bmj. 2008;336(7651):1003-1006.

9. Keane WF, Eknoyan G. Proteinuria, albuminuria, risk, assessment, detection, elimination (PARADE): a position paper of the National Kidney Foundation. American Journal of Kidney Diseases. 1999;33(5):1004-1010.
10. Turpin CA, Sakyi SA, Owiredu WK, Ephraim RK, Anto EO. Association between adverse pregnancy outcome and imbalance in angiogenic regulators and oxidative stress biomarkers in gestational hypertension and preeclampsia. BMC pregnancy and childbirth. 2015;15(1):189.

11. Palm M. Oxidative Stress, Angiogenesis and Inflammation in Normal Pregnancy and Postpartum. Acta Universitatis Upsaliensis Digital comprehensive summaries of Uppasala Dissertations from the Faculty of Medicine Uppsala ISBN 978-91-554-8314-2 2012;753-763.

12. Turpin CA, Sakyi SA, Owiredu WK, Ephraim RK, Anto EO. Association between adverse pregnancy outcome and imbalance in angiogenic regulators and oxidative stress biomarkers in gestational hypertension and preeclampsia. BMC pregnancy and childbirth 2015;15(1):1.

13. De Vivo A, Baviera G, Giordano D, Todarello G, Corrado F, D'anna R. Endoglin, PIGF and sFlt-1 as markers for predicting pre-eclampsia. Acta obstetricia et gynecologica Scandinavica 2008;87(8):837-842.

14. Hertig A, Liere P. New markers in preeclampsia. Clinica Chimica Acta. 2010;411(21):1591-1595.

15. Raymond D, Peterson E. A critical review of early-onset and late-onset preeclampsia. Obstetrical \& gynecological survey 2011;66(8):497506.

16. Kirkendall WM, Burton AC, Epstein FH, Freis ED. Recommendations for human blood pressure determination by sphygmomanometers. Circulation. 1967;36(6):980-988.

17. Pinheiro CC, Rayol P, Gozzani L, Reis LM, Zampieri G, Dias CB, et al. The relationship of angiogenic factors to maternal and neonatal manifestations of early-onset and late-onset preeclampsia. Prenatal diagnosis. 2014;34(11):1084-1092.

18. Wilkström A, Nash P, Ericsson U: Evidence of increased oxidative stress and change in the plasminogen activator inhibitor (PAI-1)-1 to PAI-2 ratio in early-onset but not late-onset preeclampsia. Am J Obstet Gynecol. 2009;201(6):e1-597.e8.

19. Unal ER, Robinson CJ, Johnson DD, Chang EY. Second-trimester angiogenic factors as biomarkers for future-onset preeclampsia. American journal of obstetrics and gynecology. 2007;197(2):211. e211-211.e214.

20. Ohkuchi A, Hirashima C, Matsubara S, Suzuki H, Takahashi K, Arai F, et al. Alterations in placental growth factor levels before and after the onset of preeclampsia are more pronounced in women with early onset severe preeclampsia. Hypertension research. 2007;30(2):151159.

21. Zeisler H, Elisa L, Frederic C, Manu V, Anne C, Maria S, et al. Predictive Value of the sFlt-1: PlGF Ratio in Women with Suspected Preeclampsia. N Engl J Med. 2016;374 (1):13-22.

22. Klein E, Schlembach D, Ramoni A, Langer E, Bahlmann F, Grill S, et al. Influence of the sFlt-1/PIGF Ratio on Clinical Decision-Making in Women with Suspected Preeclampsia. PLoS ONE 2016,11(5): e0156013. doi:10.1371/journal.pone.0156013.

23. Baba Y, Ohkuchi A, Usui R, Takahashi H, Matsubara S. Urinary protein-to-creatinine ratio indicative of significant proteinuria in normotensive pregnant women. J Obstet Gynaecol Res. 2016;42(7):784-788. 